\title{
TRATAMENTO HEPATOPROTETOR FAVORECE A RESPOSTA LEUCOCITÁRIA DE RATOS WISTAR INTOXICADOS POR $\mathrm{CCl}_{4}$
}

\author{
Hepatoprotective treatment improves leukocyte responses of Wistar rats intoxicated \\ by $\mathrm{CCl}_{4}$
}

\author{
BELO, M.A.A. ${ }^{1,2}$; SOUZA, L.M. ${ }^{1,2}$; SOARES, V. E. ${ }^{1,2}$; SOBREIRA, M.F.R. ${ }^{1}$; CASOL, \\ D.M.S. ${ }^{3}$; TOMA, S.B. ${ }^{3}$ \\ 1 Unicastelo, Descalvado/SP. \\ ${ }^{2}$ CPPAR- Depto. Patologia Veterinária - FCAV/Unesp - Campus de Jaboticabal. \\ ${ }^{3}$ Médico Veterinário, Depto. de Pesquisas Veterinárias - Empresa Ouro Fino Saúde Animal Ltda.
}

Endereço para correspondência: Marco Antonio de Andrade Belo: maabelo@hotmail.com

\begin{abstract}
RESUMO
A presente investigação teve por objetivo avaliar o efeito hepatoprotetor de tratamentos com o composto de $\mathrm{N}$ - Acetil DL- Metionina (5\%) + Cloreto de Colina (2\%) + Cafeína (1\%) + Tiamina Cloridrato (1\%) + Nicotinamida $(0,5 \%)$ + Piridoxina Cloridrato $(0,04 \%)$, administrado via Intramuscular (IM) nas doses de 0,2; 0,6 e 1,0 L/Kg de peso vivo (PV), através do estudo leucocitário em ratos Wistar, submetidos à intoxicação aguda com $\mathrm{CCl}_{4}$. Fêmeas $(\mathrm{N}=147)$ foram distribuídas aleatoriamente em 21 grupos, constituindo seis diferentes tratamentos, sendo avaliados sete animais por grupo em quatro períodos: dois, quatro, seis e oito dias pós-intoxicação induzida pelo $\mathrm{CCl}_{4}$. Verificou-se neste estudo quadros de eosinofilia e monocitose absoluta em animais não tratados e tratados com a dose mínima de $0,2 \mathrm{~mL}$, sendo estes efeitos significativamente amenizados em animais tratados com 0,6 e $1,0 \mathrm{~mL}$ do composto hepatoprotetor $/ \mathrm{kg}$ de PV. Os animais intoxicados e não tratados apresentaram um quadro de trombocitopenia, quando comparado a animais intoxicados e tratados. Houve neutropenia e linfocitose absoluta em todos os ratos intoxicados com $\mathrm{CCl}_{4}$, não existindo diferença entre os tratamentos. A análise do leucograma demonstrou que o tratamento com o composto hepatoprotetor favoreceu a resposta leucocitária, por atuar beneficamente sobre a população destas células, corroborando a hipótese de que tais eventos amenizem os efeitos deletérios da intoxicação por $\mathrm{CCl}_{4}$ no tecido hepático.
\end{abstract}

Palavras-chave: hepatoproteção; leucócitos; ratos; tetracloreto de carbono

ABSTRACT

The aims of this investigation was to evaluate the effect of hepatoprotective treatments with a compound prepared by the association of $\mathrm{N}$-Acetyl DL-Methionine $(5 \%)+$ Choline chloride $(2 \%)+$ Caffeine $(1 \%)+$ Thiamine hydrochloride (1\%) + Nicotinamida $(0,5 \%)+$ Pyridoxine hydrochloride $(0.04 \%)$, administered through intramuscular (IM) route, at doses of $0.2,0.6$ and $1.0 \mathrm{~mL} / \mathrm{kg}$ of BW, through the study of leukocytes responses in rats submitted to acute intoxication with $\mathrm{CCl}_{4}$. 147 females were randomized into 21 groups, performing five different treatments, which were evaluated seven animals in four periods: two, four, six and eight days after $\mathrm{CCl}_{4}$-induced intoxication. In this study, it was observed absolute eosinophilia and monocytosis in animals untreated and treated with the lowest dose of $0.2 \mathrm{~mL}$. These responses were significantly better in animals treated with 0.6 and $1.0 \mathrm{~mL} / \mathrm{kg}$ BW. The untreated animals showed thrombocytopenia, when compared to treated animals. Absolute neutropenia and lymphocytosis was observed in all rats intoxicated with $\mathrm{CCl} 4$, there is no difference among treatments. The analysis of white blood cells demonstrated that the hepatoprotective treatments favored the leukocyte response, by act beneficially on the population of these cells, supporting the hypothesis that these events may reduce the deleterious effects in liver tissue after intoxication by $\mathrm{CCl}_{4}$.

Key words: carbon tetrachloride; hepatoprotective; leukocytes; rats 


\section{INTRODUÇÃO}

Os animais estão continuamente expostos a diferentes tipos de substâncias potencialmente tóxicas, tais como: pesticidas, resíduos industriais, alimentos deteriorados, entre outros (Nevin e Vijayammal, 2005). A maioria destes compostos químicos induz a peroxidação de lipídios mediada pela presença de radicais livres, resultando na lesão de biomembranas, consequentemente, promovendo disfunções celulares e teciduais (Cho et al., 2003).

Os processos peroxidativos envolvendo lipídios de membrana celulares representam fatores cruciais na patogênese de doenças relacionadas aos radicais livres, resultando na ocorrência de processos inflamatórios e disfunções hepáticas (Haliwell, 1997; Datta et al., 1998). Na fisiopatologia destes processos, ocorre intensa infiltração de células inflamatórias para os tecidos lesados, agravando muitas vezes o quadro clínico do animal (Yang et al., 2006; Lin et al., 2008; Jain, et al., 2008), comprometendo os mecanismos de reparo e regeneração tecidual (Tracey, 2007).

De acordo com Macedo (2008), os leucócitos circulantes são células extremamente importantes no desenvolvimento da resposta inflamatória, participando efetivamente destes eventos. Neste contexto, a análise leucocitária sangüínea pode fornecer subsídios importantes para 0 auxílio diagnóstico e prognóstico de condições mórbidas em populações de animais (Tavares-Dias e Moraes, 2003). Por outro lado, o tetracloreto de carbono $\left(\mathrm{CCl}_{4}\right)$ é um agente químico hepatotóxico seletivo, atuando como xenobiótico na indução de estresse oxidativo, causando severas injúrias no tecido hepático (Jeon et al., 2003; Kanter et al., 2005).

Evidências indicam que nutrientes antioxidantes, tais como: vitamina $\mathrm{E}$, selênio, entre outros, podem prevenir lesões hepáticas induzidas pelo tetracloreto de carbono (Shen et al., 2005). Para Attia e Ali (2006), os níveis de fósforo, potássio, zinco, selênio, vitamina $A, C$ e complexo $B$ presentes no bulbo do alho podem ter contribuído na atividade antioxidativa em ratos intoxicados com $\mathrm{CCl}_{4}$. Estudos demonstraram que os processos de recuperação hepática são favorecidos, entre outros, pela biodisponibilidade de aminoácidos (Krause et al., 2002; Nishitani et al., 2005). Habu et al. (2003) e Nishitani et al. (2005) verificaram a participação benéfica da suplementação com aminoácidos sobre a melhora no metabolismo da glicose e albumina, respectivamente, em ratos apresentando cirrose induzida por $\mathrm{CCl}_{4}$.

Devido à importância hepatoprotetora que representa metabolicamente a atividade antioxidante e regenerativa dos nutrientes supracitados, este estudo teve por objetivo investigar o efeito do composto medicamentoso formulado com base nestes preceitos sobre a resposta leucocitária de ratos Wistar, submetidos à modelo clássico de intoxicação aguda com $\mathrm{CCl}_{4}$.

\section{MATERIAL E MÉTODOS}

Foram utilizados 147 fêmeas de ratos Wistar, pesando de 180 a 230 gramas, provenientes do biotério da Unesp de Botucatu, em concordância com protocolo experimental aprovado pelo Comitê de Ética institucional da UNICASTELO (Processo: 2277-2497/08). Foram avaliados sete animais por tratamento em quatro períodos, isto é: dois, quatro, seis e oito dias após a intoxicação induzida pela aplicação de $\mathrm{CCl}_{4}$, via subcutânea, diluído 1:1 em óleo de oliva e administrado na dose de $5 \mathrm{~mL} / \mathrm{kg}$ de peso vivo.

Os animais foram distribuídos em 6 grupos: $\mathrm{T} 1=$ não intoxicados $\mathrm{e}$ não tratados; $\mathrm{T} 2=$ intoxicados com $\mathrm{CCl}_{4}$ e não tratado; T3, T4 e T5 = intoxicados com $\mathrm{CCl}_{4}$ e tratados com as doses de $0.2,0.6$ e 1.0 $\mathrm{mL} / \mathrm{Kg}$ de peso vivo do hepatoprotetor 
injetável (Ouro Fino Saúde Animal) (cada $100 \mathrm{~mL}$ contém: N- Acetil DL- Metionina 5,00 g; Cloreto de Colina 2,00g; Cafeína 1,00g; Tiamina Cloridrato 1,00g; Nicotinamida $0,50 \mathrm{~g}$; Piridoxina Cloridrato $0,04 \mathrm{~g}$ ), respectivamente. Os tratamentos com as diferentes doses do hepatoprotetor injetável foram realizados diariamente após a intoxicação dos animais com $\mathrm{CCl}_{4}$, através da administração intramuscular no membro posterior direito, com intervalo entre doses de 24 horas. O tratamento T2 (controle negativo) foi submetido à administração de solução fisiológica a 0,9\% na dose de $0,6 \mathrm{~mL} / \mathrm{kg}$ de peso vivo, para padronização do estímulo de estresse por captura e aplicação dos medicamentos.

Os animais foram anestesiados com associação cetamina $10 \%$ e xilazina $2 \%$ na proporção de 6:1, administrada via intramuscular na dose de $1 \mathrm{~mL}$ da solução/kg de PV, para colheita de sangue da veia cava caudal, utilizando-se heparina sódica como anticoagulante. $\mathrm{Na}$ determinação do leucograma foi utilizado contador automático de células sanguíneas (Modelo CC510, CELM - Cia. Equipadora de Laboratórios Modernos) e a contagem de leucócitos e trombócitos foi realizada manualmente em câmara de Neubauer. No momento da coleta de sangue, pequenas amostras sem anticoagulante foram destinadas à confecção de extensões sangüíneas para a contagem diferencial de leucócitos. Após coloração com MayGrünwald-Giemsa-Wright, foi realizada a contagem de 200 células, estabelecendo o percentual de cada tipo celular.

Os dados experimentais foram submetidos à análise de variância através de um delineamento inteiramente casualizado em parcela subdividida no tempo, tendo como tratamentos principais os grupos experimentais (seis tratamentos) e as subparcelas o período experimental (2, 4, 6 e 8 dias) utilizando-se o pacote estatístico SAS, pelo procedimento PROC MIXED, versão 8.2 (Statistical Analysis System, 2001). As comparações múltiplas foram aferidas pelo teste T ao nível de 95\% de confiança e acordo com Snedecor e Cochran (1980).

\section{RESULTADOS}

No estudo trombocitário de animais intoxicados (Figura 1), verificou-se aumento circulante no número de plaquetas no segundo dia e trombocitopenia no oitavo dia após a administração do toxicante, sendo que estes resultados não apresentaram significância estatística. Enquanto, o estudo leucocitário demonstrou elevado número de leucócitos totais no quarto e sexto dias, sendo seguido por leucopenia no oitavo dia do processo patológico, não ocorrendo diferença significativa $(P>0,05)$ na observação destes resultados (Figura 1).

$\mathrm{Na}$ contagem diferencial de leucócitos, não se observou variações significativas $(P>0,05)$ no número absoluto e relativo de neutrófilos segmentados circulantes na comparação entre animais submetidos ou não a intoxicação, dois dias após a aplicação do $\mathrm{CCl}_{4}$ (Figura 2 e Tabela 1, respectivamente). Porém, nesta mesma análise, ratos tratados com 0,2 e $0,6 \mathrm{~mL}$ do protetor hepático apresentaram neutropenia relativa e absoluta em relação aos animais tratados com $1,0 \mathrm{~mL}$ do hepatoprotetor $/ \mathrm{kg}$ de PV.

No quarto dia de evolução do quadro clínico de intoxicação, constatou-se diminuição significativa $(P<0,05)$ no número absoluto e relativo de neutrófilos segmentados em animais que foram submetidos ao toxicante quando comparados ao grupo de ratos não intoxicados (T1), permanecendo relativamente baixa $(P<0,05)$ a contagem celular no sexto dia, não existindo variações leucocitárias para este parâmetro entre os animais intoxicados (Figura 2 e Tabela 1).

Os ratos tratados com $0,6 \mathrm{~mL}$ (T4) demonstraram neutropenia absoluta e relativa no oitavo dia, no entanto, a contagem de neutrófilos bastonetes (Figura 2 e Tabela 1) revelou aumento no número destas células neste mesmo período, indi- 
(A)

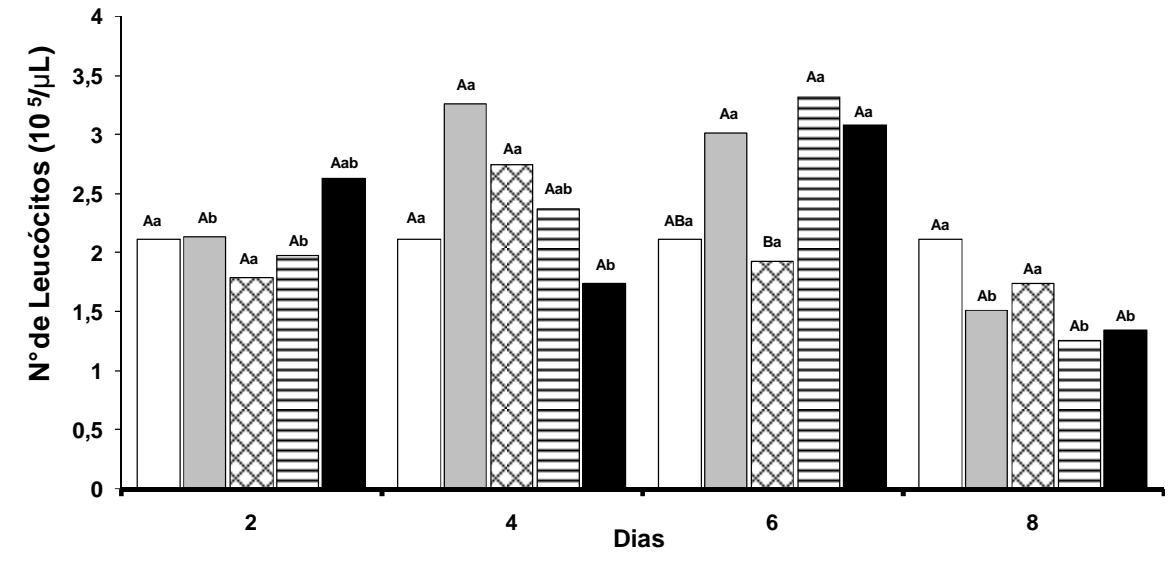

(B)

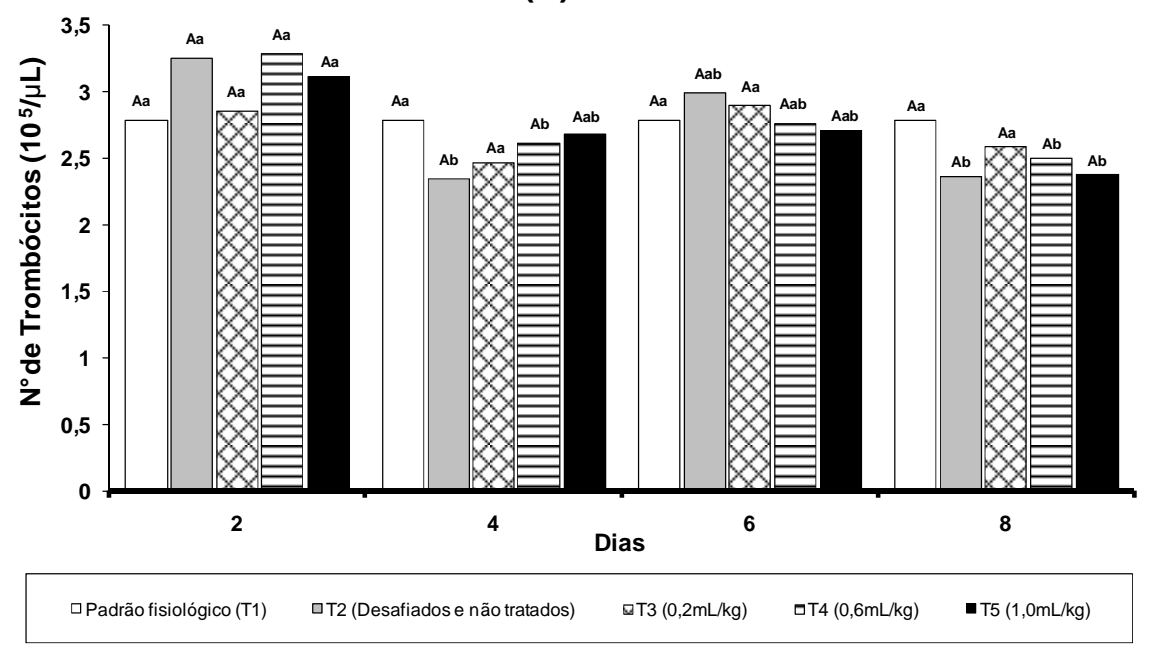

Figura 1 - Contagem de leucócitos totais (A) e trombócitos (B) aferidos em ratos Wistar submetidos aos diferentes tratamentos, após intoxicação com $\mathrm{CCl}_{4}$

Médias seguidas pela mesma letra não diferem entre si pelo teste $T(P \geq 0,05)$,onde letra maiúscula compara tratamentos em cada dia analisado, e minúscula compara cada tratamento nos dias analisados.

$\mathrm{T} 1=$ sem tratamento e sem desafio com o $\mathrm{CCl}_{4} ; \mathrm{T} 2=\mathrm{Sem}$ tratamento e desafiado com $\mathrm{CCl}_{4} ; \mathrm{T} 3$, T4 e T5 = tratado com doses de 0,2; 0,6 e $1,0 \mathrm{~mL} / \mathrm{kg}$ e desafiado com $\circ \mathrm{CCl}_{4}$, respectivamente.

cando a recuperação plasmática deste leucócito.

Animais tratados com $1,0 \mathrm{~mL}$ do hepatoprotetor (T5) apresentaram monocitose absoluta e relativa $(P<0,05)$ em relação aos ratos não intoxicados $(T 1)$, no segundo dia após a indução de hepatotoxicidade pelo $\mathrm{CCl}_{4}$. Contudo, no quarto dia, verificou-se monocitose em todos os animais intoxicados, exceto aque- les submetidos ao tratamento com $1,0 \mathrm{~mL}$ do antitóxico $/ \mathrm{kg}$ do PV. Neste período, o aumento relativo e absoluto na contagem de monócitos foi mais expressiva em ratos intoxicados e não tratados (T2).

No estudo linfocitário, verificou-se a ocorrência de linfocitose absoluta e relativa no quarto e sexto dias, sendo significativo 0 aumento $(P<0,05)$ no número de linfócitos apenas nos animais tratados com $0,2 \mathrm{~mL}$ 

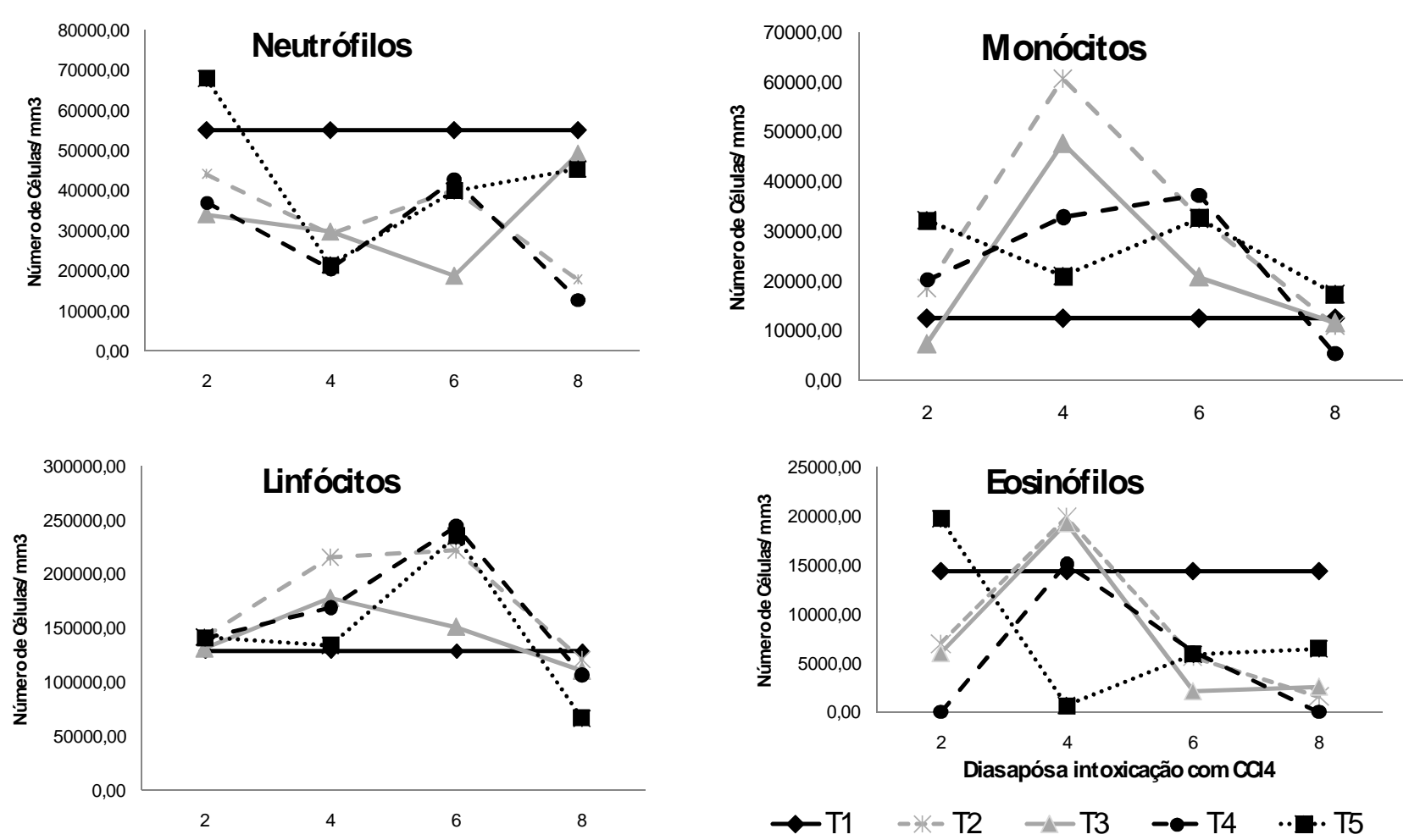

Figura 2 - Contagem diferencial de leucócitos absoluta aferidos em ratos Wistar submetidos aos tratamentos, após intoxicação com $\mathrm{CCl}_{4} . \mathrm{T} 1=$ sem tratamento e sem desafio com o $\mathrm{CCl}_{4} ; \mathrm{T} 2=\mathrm{Sem}$ tratamento e desafiado com $\mathrm{CCl}_{4} ; \mathrm{T} 3$, T4 e T5 = tratado com doses de 0,2;0,6 e 1,0 mL/kg e desafiado com o $\mathrm{CCl}_{4}$, respectivamente.

do protetor hepático quando comparados aos ratos não intoxicados (Figura $2 \mathrm{e}$ Tabela 1, respectivamente). No entanto, a contagem absoluta de linfócitos apresentou aumento significativo $(P<0,05)$ nas concentrações circulantes seis dias após a indução do quadro de intoxicação, principalmente, para animais tratados com 0,6 e 1,0 mL do hepatoprotetor/kg de PV.

No oitavo dia de evolução do quadro clínico, constatou-se normalização para as contagens absolutas de linfócitos em todos os grupos (Figura 2), ocorrendo apenas linfocitose relativa no grupo tratado com 0,6 $\mathrm{mL}$ do composto medicamentoso (Tabela 1). Porém, estes resultados não apresentaram significância quando confrontados com os valores absolutos observados neste mesmo grupo.

No estudo leucocitário, verificou-se níveis normais nas contagens absolutas e relativas de eosinófilos em animais tratados com 1,0 mL do hepatoprotetor, enquanto, os demais grupos submetidos a intoxicação com $\mathrm{CCl}_{4}$ (T2, T3 e T4) apresentaram eosinopenia significativa $\quad(P<0,0001)$ quando comparados aos ratos não intoxicados, dois dias após a administração do toxicante (Figura 2 e Tabelas 1). Entretanto, no quarto dia, ocorreu diminuição significativa $(P<0,0001)$ no número absoluto e relativo de eosinófilos circulantes nos animais tratados com 1,0 $\mathrm{mL}$ do antitóxico/kg de P.V. se comparados aos outros tratamentos. O quadro clínico de eosinopenia se manteve significativo até o oitavo dia. Por outro lado, não se observou variações nas contagens relativas e absolutas de basófilos, resultante do pequeno número de células presentes nas extensões sangüíneas.

\section{DISCUSSÃO}

Ohta et al (2006) demonstraram o efeito hepatoprotetor da neutropenia causada pela administração de antisoro antineutrófilo sobre a diminuição da resposta 
Tabela 1 - Valores médios com respectivos desvios-padrão e análise de variância ${ }^{1}$ da contagem de leucócitos relativa para neutrófilos segmentados e bastonetes, monócitos, linfócitos, eosinófilos e basófilos aferidos em ratos Wistar, submetidos à intoxicação com $\mathrm{CCl}_{4}$

\begin{tabular}{|c|c|c|c|c|c|c|c|c|c|c|c|c|c|c|c|c|c|c|c|c|c|c|c|}
\hline \multirow{2}{*}{ 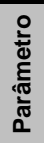 } & \multirow{2}{*}{$\begin{array}{l}\text { Período } \\
\text { (dias) }\end{array}$} & \multicolumn{20}{|c|}{ Tratamentos $^{2}$} & \multicolumn{2}{|c|}{ Desdobramento } \\
\hline & & \multicolumn{4}{|c|}{ T1 } & \multicolumn{4}{|c|}{ T2 } & \multicolumn{4}{|c|}{ T3 } & \multicolumn{4}{|c|}{ T4 } & \multicolumn{4}{|c|}{ T5 } & \multirow{2}{*}{$\begin{array}{c}\begin{array}{c}\text { Valor } \\
\text { de F }\end{array} \\
5,44\end{array}$} & \multirow{2}{*}{$\begin{array}{c}\text { Pr>F } \\
0,0001\end{array}$} \\
\hline \multirow{4}{*}{ 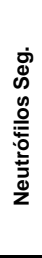 } & 2 & 25,90 & \pm & 2,96 & $\begin{array}{l}A B \\
a\end{array}$ & 20,71 & \pm & 9,16 & $\begin{array}{l}A B \\
b\end{array}$ & 18,43 & \pm & 11,91 & $\begin{array}{l}B \\
a b\end{array}$ & 18,00 & \pm & 11,42 & $\begin{array}{l}\text { B } \\
\text { a }\end{array}$ & 27,43 & \pm & 12,80 & $\begin{array}{l}A B \\
a\end{array}$ & & \\
\hline & 4 & 25,90 & \pm & 2,96 & $\begin{array}{l}A \\
a\end{array}$ & 9,00 & \pm & 1,29 & $\begin{array}{l}\text { B } \\
\text { b }\end{array}$ & 10,86 & \pm & 1,21 & $\begin{array}{l}\text { B } \\
\text { b }\end{array}$ & 9,29 & \pm & 2,69 & $\begin{array}{l}\text { B } \\
\text { a }\end{array}$ & 12,29 & \pm & 4,86 & $\begin{array}{l}\text { B } \\
\text { b }\end{array}$ & 7,75 & $<0,0001$ \\
\hline & 6 & 25,90 & \pm & 2,96 & $\begin{array}{l}\text { A } \\
a\end{array}$ & 13,00 & \pm & 2,89 & $\begin{array}{l}\text { B } \\
\text { b }\end{array}$ & 10,00 & \pm & 1,83 & $\begin{array}{l}\text { B } \\
\text { b }\end{array}$ & 12,71 & \pm & 3,77 & $\begin{array}{l}\text { B } \\
\text { a }\end{array}$ & 13,43 & \pm & 4,20 & $\begin{array}{l}\text { B } \\
\text { b }\end{array}$ & 6,16 & $<0,0001$ \\
\hline & 8 & 25,90 & \pm & 2,96 & $\begin{array}{l}\mathrm{AB} \\
\mathrm{a}\end{array}$ & 15,43 & \pm & 15,39 & $\begin{array}{l}\text { BC } \\
\text { b }\end{array}$ & 27,71 & \pm & 10,06 & $\begin{array}{l}\mathrm{AB} \\
\mathrm{a} \\
\end{array}$ & 10,57 & \pm & 2,88 & $\begin{array}{l}\mathrm{C} \\
\mathrm{a} \\
\end{array}$ & 32,57 & \pm & 8,08 & $\begin{array}{l}\text { A } \\
\mathbf{a}\end{array}$ & 13,6 & $<0,0001$ \\
\hline \multicolumn{2}{|c|}{$\begin{array}{c}\text { Valor de } \mathrm{F} \\
\mathrm{Pr}>\mathrm{F}\end{array}$} & $\begin{array}{r}0,00 \\
1,0000 \\
\end{array}$ & & & & $\begin{array}{r}3,55 \\
0,0158 \\
\end{array}$ & & & & $\begin{array}{r}10,04 \\
<0,0001 \\
\end{array}$ & & & & $\begin{array}{r}2,19 \\
0,0919 \\
\end{array}$ & & & & $\begin{array}{r}15,20 \\
<0,0001 \\
\end{array}$ & & & & & \\
\hline \multirow{4}{*}{ 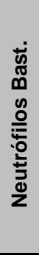 } & 2 & 0,00 & \pm & 0,00 & $\begin{array}{l}\text { A } \\
\mathbf{a}\end{array}$ & 0,43 & \pm & 1,13 & $\begin{array}{l}\text { A } \\
\mathbf{a}\end{array}$ & 0,00 & \pm & 0,00 & $\begin{array}{l}\text { A } \\
\mathbf{a}\end{array}$ & 0,00 & \pm & 0,00 & $\begin{array}{l}\text { A } \\
\mathbf{a}\end{array}$ & 0,00 & \pm & 0,00 & $\begin{array}{l}\text { A } \\
\mathbf{a}\end{array}$ & 1,10 & 0,3639 \\
\hline & 4 & 0,00 & \pm & 0,00 & $\begin{array}{l}\text { A } \\
\mathbf{a}\end{array}$ & 0,14 & \pm & 0,38 & $\begin{array}{l}\text { A } \\
\text { a }\end{array}$ & 0,00 & \pm & 0,00 & $\begin{array}{l}\text { A } \\
\text { a }\end{array}$ & 0,29 & \pm & 0,76 & $\begin{array}{l}\text { A } \\
\mathbf{a}\end{array}$ & 0,00 & \pm & 0,00 & $\begin{array}{l}\text { A } \\
\text { a }\end{array}$ & 0,52 & 0,7609 \\
\hline & 6 & 0,00 & \pm & 0,00 & $\begin{array}{l}\text { A } \\
\text { a }\end{array}$ & 0,00 & \pm & 0,00 & $\begin{array}{l}\text { A } \\
\mathbf{a}\end{array}$ & 0,00 & \pm & 0,00 & $\begin{array}{l}\text { A } \\
\mathbf{a}\end{array}$ & 0,14 & \pm & 0,38 & $\begin{array}{l}\text { A } \\
\mathbf{a}\end{array}$ & 0,14 & \pm & 0,38 & $\begin{array}{l}\text { A } \\
\mathbf{a}\end{array}$ & 0,20 & 0,9625 \\
\hline & 8 & 0,00 & \pm & 0,00 & $\begin{array}{l}\mathbf{A} \\
\mathbf{a}\end{array}$ & 0,00 & \pm & 0,00 & $\begin{array}{l}\text { A } \\
\mathbf{a}\end{array}$ & 0,29 & \pm & 0,76 & $\begin{array}{l}\text { A } \\
\mathbf{a}\end{array}$ & 0,57 & \pm & 1,51 & $\begin{array}{l}\text { A } \\
\mathbf{a}\end{array}$ & 0,00 & \pm & 0,00 & $\begin{array}{l}\text { A } \\
\mathbf{a}\end{array}$ & 2,08 & 0,0707 \\
\hline \multicolumn{2}{|c|}{$\begin{array}{c}\text { Valor de } \mathrm{F} \\
\mathrm{Pr}>\mathrm{F}\end{array}$} & $\begin{array}{r}1,44 \\
0,2320 \\
\end{array}$ & & & & $\begin{array}{r}0,72 \\
0,5401 \\
\end{array}$ & & & & $\begin{array}{r}2,11 \\
0,1016 \\
\end{array}$ & & & & $\begin{array}{r}0,18 \\
0,9095 \\
\end{array}$ & & & & $\begin{array}{r}0,00 \\
1,0000 \\
\end{array}$ & & & & & \\
\hline \multirow{5}{*}{$\begin{array}{l}00 \\
\stackrel{0}{0} \\
0 \\
0 \\
0 \\
\Sigma\end{array}$} & 2 & 5,80 & \pm & 1,23 & $\begin{array}{l}\text { B } \\
\text { a }\end{array}$ & 8,71 & \pm & 4,79 & $\begin{array}{l}A B \\
b\end{array}$ & 4,29 & \pm & 1,70 & $\begin{array}{l}\text { B } \\
\text { C }\end{array}$ & 10,14 & \pm & 2,97 & $\begin{array}{l}A B \\
a b\end{array}$ & 11,86 & \pm & 4,45 & $\begin{array}{l}\text { A } \\
\mathbf{a}\end{array}$ & 5,82 & $<0,0001$ \\
\hline & 4 & 5,80 & \pm & 1,23 & $\begin{array}{l}\text { B } \\
\text { a }\end{array}$ & 18,14 & \pm & 4,78 & $\begin{array}{l}\text { A } \\
\mathbf{a}\end{array}$ & 16,86 & \pm & 5,79 & $\begin{array}{l}\text { A } \\
\text { a }\end{array}$ & 13,57 & \pm & 2,76 & $\begin{array}{l}\text { A } \\
\mathbf{a}\end{array}$ & 12,14 & \pm & 4,41 & $\begin{array}{l}\text { A } \\
\mathbf{a}\end{array}$ & 15,73 & $<0,0001$ \\
\hline & 6 & 5,80 & \pm & 1,23 & $\begin{array}{l}\mathrm{B} \\
\mathrm{a}\end{array}$ & 10,14 & \pm & 3,44 & $\begin{array}{l}\mathrm{AB} \\
\mathrm{b}\end{array}$ & 10,86 & \pm & 3,72 & $\begin{array}{l}A B \\
a b\end{array}$ & 10,71 & \pm & 3,15 & $\begin{array}{l}A B \\
a b\end{array}$ & 10,57 & \pm & 3,31 & $\begin{array}{l}A B \\
a\end{array}$ & 3,93 & 0,0022 \\
\hline & 8 & 5,80 & \pm & 1,23 & $\begin{array}{l}\text { B } \\
\text { a }\end{array}$ & 7,29 & \pm & 2,93 & $\begin{array}{l}A B \\
b\end{array}$ & 6,57 & \pm & 2,15 & $\begin{array}{l}A B \\
b c\end{array}$ & 4,43 & \pm & 1,27 & $\begin{array}{l}\text { B } \\
\text { b }\end{array}$ & 12,29 & \pm & 4,39 & $\begin{array}{l}\text { A } \\
\text { a }\end{array}$ & 4,98 & 0,0003 \\
\hline & $\begin{array}{l}\text { alor de } F \\
\operatorname{Pr}>F\end{array}$ & $\begin{array}{r}0,00 \\
1,0000 \\
\end{array}$ & & & & $\begin{array}{r}15,94 \\
<0,0001 \\
\end{array}$ & & & & $\begin{array}{r}20,64 \\
<0,0001 \\
\end{array}$ & & & & $\begin{array}{r}9,91 \\
<0,0001 \\
\end{array}$ & & & & $\begin{array}{r}0,41 \\
0,7434 \\
\end{array}$ & & & & & \\
\hline \multirow{4}{*}{ 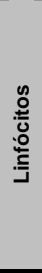 } & 2 & 61,30 & \pm & 4,14 & $\begin{array}{l}A B \\
a\end{array}$ & 67,29 & \pm & 11,35 & $\begin{array}{l}A B \\
a\end{array}$ & 74,43 & \pm & 12,01 & $\begin{array}{l}A \\
a b\end{array}$ & 71,86 & \pm & 12,80 & $\begin{array}{l}\text { A } \\
\text { ab }\end{array}$ & 53,57 & \pm & 8,20 & $\begin{array}{l}\text { B } \\
\text { b }\end{array}$ & 7,02 & $<0,0001$ \\
\hline & 4 & 61,30 & \pm & 4,14 & $\begin{array}{l}\text { B } \\
\text { a }\end{array}$ & 67,00 & \pm & 5,69 & $\begin{array}{l}A B \\
a\end{array}$ & 65,29 & \pm & 5,59 & $\begin{array}{l}A B \\
a b\end{array}$ & 70,14 & \pm & 4,53 & $\begin{array}{l}A B \\
a b\end{array}$ & 75,86 & \pm & 7,34 & $\begin{array}{l}\text { A } \\
\mathbf{a}\end{array}$ & 3,67 & 0,0036 \\
\hline & 6 & 61,30 & \pm & 4,14 & $\begin{array}{l}\text { B } \\
\text { a }\end{array}$ & 75,00 & \pm & 5,97 & $\begin{array}{l}A B \\
a\end{array}$ & 77,86 & \pm & 4,53 & $\begin{array}{l}\text { A } \\
\mathbf{a}\end{array}$ & 74,57 & \pm & 5,71 & $\begin{array}{l}A B \\
a b\end{array}$ & 76,00 & \pm & 6,22 & $\begin{array}{l}\text { A } \\
\mathbf{a}\end{array}$ & 5,20 & 0,0002 \\
\hline & 8 & 61,30 & \pm & 4,14 & $\begin{array}{l}\mathrm{C} \\
\mathrm{a}\end{array}$ & 76,00 & \pm & 17,01 & $\begin{array}{l}\mathrm{AB} \\
\mathrm{a}\end{array}$ & 63,71 & \pm & 10,37 & $\begin{array}{l}\text { BC } \\
\text { b }\end{array}$ & 84,43 & \pm & 4,76 & $\begin{array}{l}\text { A } \\
\text { a }\end{array}$ & 51,43 & \pm & 11,80 & $\begin{array}{l}\text { C } \\
\text { b }\end{array}$ & 18,60 & $<0,0001$ \\
\hline \multicolumn{2}{|c|}{$\begin{array}{c}\text { Valor de } \mathrm{F} \\
\mathrm{Pr}>\mathrm{F}\end{array}$} & $\begin{array}{r}0,00 \\
1,0000\end{array}$ & & & & $\begin{array}{r}2,64 \\
0,0518\end{array}$ & & & & $\begin{array}{r}5,34 \\
0,0016\end{array}$ & & & & $\begin{array}{r}4,58 \\
0,0042\end{array}$ & & & & $\begin{array}{r}20,64 \\
<0,0001 \\
\end{array}$ & & & & & \\
\hline \multirow{5}{*}{ 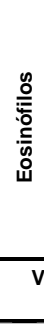 } & 2 & 7,00 & \pm & 1,63 & $\begin{array}{l}\text { A } \\
\mathrm{a}\end{array}$ & 2,71 & \pm & 2,87 & $\begin{array}{l}\mathrm{BC} \\
\mathrm{ab}\end{array}$ & 2,86 & \pm & 4,78 & $\begin{array}{l}\mathrm{BC} \\
\mathrm{ab}\end{array}$ & 0,00 & \pm & 0,00 & $\begin{array}{l}\text { C } \\
\text { b }\end{array}$ & 6,86 & \pm & 5,98 & $\begin{array}{l}A B \\
a\end{array}$ & 13,74 & $<0,0001$ \\
\hline & 4 & 7,00 & \pm & 1,63 & $\begin{array}{l}\text { A } \\
\mathbf{a}\end{array}$ & 5,71 & \pm & 3,04 & $\begin{array}{l}\mathbf{A} \\
\mathbf{a}\end{array}$ & 7,00 & \pm & 3,16 & $\begin{array}{l}\mathbf{A} \\
\mathbf{a}\end{array}$ & 7,43 & \pm & 3,51 & $\begin{array}{l}\text { A } \\
\mathbf{a}\end{array}$ & 0,43 & \pm & 0,79 & $\begin{array}{l}\text { B } \\
\text { b }\end{array}$ & 13,41 & $<0,0001$ \\
\hline & 6 & 7,00 & \pm & 1,63 & $\begin{array}{l}\text { A } \\
\mathbf{a}\end{array}$ & 1,86 & \pm & 1,86 & $\begin{array}{l}\text { B } \\
\text { ab }\end{array}$ & 1,29 & \pm & 1,38 & $\begin{array}{l}\text { B } \\
\text { b }\end{array}$ & 1,86 & \pm & 1,57 & $\begin{array}{l}\text { B } \\
\text { b }\end{array}$ & 2,00 & \pm & 1,83 & $\begin{array}{l}\text { B } \\
\text { b }\end{array}$ & 8,15 & $<0,0001$ \\
\hline & 8 & 7,00 & \pm & 1,63 & $\begin{array}{l}\text { A } \\
\mathbf{a}\end{array}$ & 1,29 & \pm & 1,50 & $\begin{array}{l}\mathrm{BC} \\
\mathrm{ab}\end{array}$ & 1,71 & \pm & 1,11 & $\begin{array}{l}\text { BC } \\
\text { b }\end{array}$ & 0,00 & \pm & 0,00 & $\begin{array}{l}\text { C } \\
\text { b }\end{array}$ & 4,57 & \pm & 1,51 & $\begin{array}{l}A B \\
a b\end{array}$ & 10,15 & $<0,0001$ \\
\hline & $\begin{array}{l}\text { alor de } \mathrm{F} \\
\mathrm{Pr}>\mathrm{F}\end{array}$ & $\begin{array}{r}0,00 \\
1,0000 \\
\end{array}$ & & & & $\begin{array}{r}4,91 \\
0,0027 \\
\end{array}$ & & & & $\begin{array}{r}8,62 \\
<0,0001 \\
\end{array}$ & & & & $\begin{array}{r}15,64 \\
<0,0001 \\
\end{array}$ & & & & $\begin{array}{r}10,17 \\
<0,0001 \\
\end{array}$ & & & & & \\
\hline \multirow{4}{*}{ 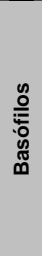 } & 2 & 0,00 & \pm & 0,00 & $\begin{array}{l}\text { A } \\
\text { a }\end{array}$ & 0,14 & \pm & 0,38 & $\begin{array}{l}\text { A } \\
\text { a }\end{array}$ & 0,00 & \pm & 0,00 & $\begin{array}{l}\text { A } \\
\text { a }\end{array}$ & 0,00 & \pm & 0,00 & $\begin{array}{l}\text { A } \\
\text { a }\end{array}$ & 0,29 & \pm & 0,76 & $\begin{array}{l}\text { A } \\
\text { a }\end{array}$ & 2,67 & 0,2238 \\
\hline & 4 & 0,00 & \pm & 0,00 & $\begin{array}{l}\text { A } \\
\mathbf{a}\end{array}$ & 0,00 & \pm & 0,00 & $\begin{array}{l}\text { A } \\
\mathbf{a}\end{array}$ & 0,00 & \pm & 0,00 & $\begin{array}{l}\text { A } \\
\mathbf{a}\end{array}$ & 0,00 & \pm & 0,00 & $\begin{array}{l}\text { A } \\
\mathbf{a}\end{array}$ & 0,00 & \pm & 0,00 & $\begin{array}{l}\text { A } \\
\mathbf{a}\end{array}$ & 0,00 & 1,0000 \\
\hline & 6 & 0,00 & \pm & 0,00 & $\begin{array}{l}\text { A } \\
\text { a }\end{array}$ & 0,00 & \pm & 0,00 & $\begin{array}{l}\text { A } \\
\mathbf{a}\end{array}$ & 0,00 & \pm & 0,00 & $\begin{array}{l}\text { A } \\
\mathbf{a}\end{array}$ & 0,14 & \pm & 0,38 & $\begin{array}{l}\text { A } \\
\mathbf{a}\end{array}$ & 0,00 & \pm & 0,00 & $\begin{array}{l}\text { A } \\
\mathbf{a}\end{array}$ & 1,02 & 0,4058 \\
\hline & 8 & 0,00 & \pm & 0,00 & $\begin{array}{l}\text { A } \\
\mathbf{a}\end{array}$ & 0,00 & \pm & 0,00 & $\begin{array}{l}\text { A } \\
\mathbf{a}\end{array}$ & 0,00 & \pm & 0,00 & $\begin{array}{l}\text { A } \\
\mathbf{a}\end{array}$ & 0,00 & \pm & 0,00 & $\begin{array}{l}\text { A } \\
\mathbf{a}\end{array}$ & 0,00 & \pm & 0,00 & $\begin{array}{l}\text { A } \\
\mathbf{a}\end{array}$ & 0,00 & 1,0000 \\
\hline \multicolumn{2}{|c|}{ Valor de F } & 0,00 & & & & 0,93 & & & & 0,00 & & & & 0,93 & & & & 3,71 & & & & & \\
\hline & $\mathrm{Pr}>\mathrm{F}$ & 1,0000 & & & & 0,4285 & & & & 1,0000 & & & & 0,4285 & & & & 0,1129 & & & & & \\
\hline
\end{tabular}


inflamatória no tecido de ratos com intoxicação aguda induzida pelo $\mathrm{CCl}_{4}$. Para estes autores, a diminuição do processo inflamatório representa uma importante forma de conter a severidade das agressões ocorridas no tecido hepático. Neste estudo, observou-se neutropenia absoluta e relativa em todos os animais intoxicados com $0 \mathrm{CCl}_{4}$, provavelmente, resultante da migração destas células para o tecido hepático lesado.

$\mathrm{O}$ metabolismo hepático do $\mathrm{CCl}_{4}$ libera o radical triclorometil $\left(\mathrm{CCl}_{3}^{-}\right)$, sendo subseqüentemente convertido em um radical peroxil na presença do oxigênio, iniciando a peroxidação de fosfolipídios da membrana citoplasmática, promovendo mudanças funcionais e morfológicas por acúmulos de produtos derivados deste processo oxidativo, causando severas injúrias nos tecidos hepáticos, como: hepatites, esteatoses, necroses centrolobulares, fibroses, cirroses e hepatomas (Poli et al., 1987; Recknagel et al., 1998). Segundo Kerr (2003), processos de lesão tecidual levam a degranulação de mastócitos e liberação de histamina, que é quimiotática para eosinófilos e estes são atraídos da medula óssea para a circulação. Observaram-se neste estudo, quadros de eosinofilia absoluta em animais intoxicados e não tratados e tratados com a dose mínima de 0,2 $\mathrm{mL}$, sugerindo a hipótese de que 0 tratamento com 0 composto hepatoprotetor nas doses de $0,6 \mathrm{~mL}$ e $1,0 \mathrm{~mL} / \mathrm{kg}$ de PV tenha auxiliado na contenção de eventos que participam diretamente dos processos lesivos em evolução no tecido hepático.

Corroborando com estes achados, verificou-se monocitose absoluta mais significativa em animais intoxicados e não tratados e tratados com a dose mínima de $0,2 \mathrm{~mL}$. Os monócitos circulantes são quimiotaticamente atraídos para o foco inflamatório, diferenciando-se em macrófagos após o processo de diapedese (Mariano e Spector, 1974). Otagawa et al. (2007) observaram favorecimento na deposição de derivados de ferro após processo de eritrofagocitose por macrófagos nos tecidos hepáticos resultante de estresse oxidativo e inflamatório. De acordo com estes autores, estes resíduos acumulados no tecido hepático participam na patogênese da esteato-hepatite.

No processo de fibrose decorrente de lesões hepáticas agudas induzidas por $\mathrm{CCl}_{4}$, a diminuição da população de linfócitos $\mathrm{B}$ circulantes atenua o processo lesivo nestes tecidos (Novobrantseva et al., 2005). Neste ensaio, ratos intoxicados com $\mathrm{CCl}_{4}$ apresentaram linfocitose absoluta entre o quarto e sexto dias, retornando para valores normais no oitavo dia, porém não existindo diferença entre os tratamentos.

Animais com lesões hepáticas podem apresentar alterações funcionais no número e função das plaquetas. Segundo Shih et al. (2005), em casos de desvios circulatórios intra e extra-hepáticos, os pacientes podem apresentar esplenomegalia congestiva, resultante da hipertensão portal, levando a quadros de trombocitopenia. Os animais não tratados e intoxicados apresentaram trombocitopenia durante a evolução do quadro patológico, sendo estatisticamente significativo a partir do quarto dia. Tal efeito ocorreu apenas no oitavo dia para os animais tratados com o composto hepatoprotetor.

\section{CONCLUSÃO}

O tratamento com o composto hepatoprotetor, baseado na suplementação com aminoácido metionina e vitaminas do complexo B, favoreceu a resposta leucocitária de ratos Wistar, por atuar beneficamente sobre as populações destas células, principalmente, controlando quadros de monocitose e eosinofilia em animais intoxicados, corroborando a hipótese de que tais eventos amenizem os efeitos deletérios do processo inflamatório, resultante da ação lesiva do $\mathrm{CCl}_{4}$ no tecido hepático. 


\section{AGRADECIMENTOS}

Os autores agradecem a Cleber Rafael Guiaro, Ed Jonnhy Rosa Prado, Rosângela Rodrigues de Souza, José Sérgio Traldi Júnior e Luiz Augusto Cavichioli por auxiliarem tecnicamente durante a realização do ensaio experimental. Esta pesquisa foi financiada pela empresa Ouro Fino Saúde Animal Ltda. e pela Universidade Camilo Castelo Branco UNICASTELO.

\section{REFERÊNCIAS}

ATTIA, M.N.T.; ALI, M.A. Hepatoprotective activity of allicin against carbon tetrachloride induced hepatic injury in rats. Journal of Biological Sciences, v.6, n.3, p. 457-468, 2006.

CHO, E.J.; YOKOZAWA, T.; RHYU, D.Y. et al. The inhibitory effects of 12 medicinal plants and their component compounds on lipid peroxidation. American Journal of Chinese Medicine, v.31, p.970, 2003.

DATTA, S.; BASU, K.; SINHA, $S$. et al. Hepatoprotective effect of a protein isolated from Cajanus indicus (Spreng) on carbon tetrachloride induced hepatotoxicity. Indian Journal of Experimental Biology, v.36, p.175-181, 1998.

HABU, D.; NISHIGUCHI, S.; NAKATANI, S. et al. Effect of oral supplementation with branched-chain amino acid granules on serum albumin level in the early stage of cirrhosis: a randomized pilot trial. Hepathology Research, v.25, p.213-318, 2003.

HALLIWEL, B. Antioxidants and human disease: a general introduction. Nutrition Review , v.55, p.4452, 1997.

JAIN, A.; SONI, M.; DEB, L. et al. Antioxidant and hepatoprotective activity of thanolic and aqueous extracts of Monordica dioica leaves. Journal of Ethanopharmacology, v.115, p.61-66, 2008.

JEON, T.I.; HWANG, S.G.; PARK, N.G. et al. Antioxidative effect of chitosan on chronic carbon tetrachloride induced hepatic injury in rats. Toxicology, v.187, n.1, p.67-73, 2003.

KERR, M.G. Glóbulos Brancos (Leucócitos). In:

Exames laboratoriais em Medicina Veterinária. Bioquímica clínica e hematologia. 2.ed. São Paulo: Roca, 2003. Cap.3, p.61-80.

KANTER, M.; COSKUN, O.; BUDANCAMANAK, M. Hepatoprotective effects of Nigella sativa $L$ and Urtica dioica $\mathrm{L}$ on lipid peroxidadtion, antioxidant enzyme systems and liver enzymes in carbo tetrachloride-treated rats. World Journal of Gastroenterology, v.11, n. 42, p.6684-6688, 2005.

KRAUSE, U.; BERTRAND, L.; MAISIN, L. et al. Signalling pathways and combinatory effects of insulin and amino acids in isolated rat hepatocytes. European Journal of Biochemistry, v. 239, p. 3742-3750, 2002.

LIN, H.M.; TSENG, H.S.; WANG, C.J. et al. Hepatoprotective effects of Solanum nigrum Linn extract against $\mathrm{CCl} 4$-induced oxidative damage in rats. Chemico-Biological Interactions, v.171, p.283-293, 2008.

MACEDO, S.M.D. Estudo dos mecanismos de ação da hidroquinona e fenol sobre o recrutamento leucocitário em respostas inflamatórias. 2008. São Paulo, 167f. Tese (Doutorado). Programa de Pósgraduação em Toxicologia e Análises Toxicológicas, Faculdade de Ciências Farmacêuticas, Universidade de São Paulo.

MARIANO, M., SPECTOR., W.G. The formation and properties of macrophage polykaryons (inflammatory giant cells). Journal of Pathology, v.113, n.1, p.119, 1974.

NEVIN, K.G.; VIJAYAMMAL, P.L. Effect of Aerva lanata against hepatotoxicity of carbon tetrachloride in rats. Environmental Toxicology and Pharmacology, v.20, p.471-477, 2005.

NISHITANI, S.; TAKEHANA, K.; FUJITANI, S. et al. Branched-chain amino acids improbé glucose metabolism in rats with liver cirrosis. American Journal of Physiology - Gastrointestal and Liver Physiology, v.288, p.1292-1300, 2005.

NOVOBRANTSEVA, T.L.; MAJEAU, G.R.; AMATUCCI, A. et al. Attenuated liver fibrosis in the absence of $B$ cells. Journal of Clinical Investigation, v.115, 3072-3082, 2005.

OHTA, Y.; IMAI, Y.; MATSURA, T. et al. Preventive effect of neutropenia on carbon tetrachloride-induced hepatotoxicity in rats. Journal of Applied Toxicology, v.26, p.178-186, 2006.

OTAGAWA, K.; KINOSHITA, K.; FUJII, H. et al. Erythrophagocytosis by liver macrophages (kupffer Cells) promotes oxidative stress, inflammation and fibrosis in a rabbit model of steatohepatitis. Gastrointestinal, Hepatobiliary and Pancreatic Pathology, v.270, n.3, p.967-980, 2007.

POLI, G.; ALBANO, E.; DIANZANI, M.U. The role of lipid peroxidation in liver damage. Chemistry and Physiology Lipids, v.45, p.117-142, 1987.

RECKNAGEL, R.O.; GLENDE, E.A. Jr.; DOLAK, J.A. et al. Mechanisms of carbon tetrachloride toxicity. 
Pharmacology and Therapeutics, v.43, p.139-154, 1998.

SAS Institute Inc. SAS/STAT software changes and enhancements though computer program. Release 8.2. Cary: SAS Institute; 2001.

SHEN, X.H.; CHENG, W.F.; LI, X.H. et al. Effects of dietary supplementation with vitamin $E$ and selenium on rat hepatic stellate cell apoptosis. World Journal of Gastroenterology, v.11, n. 32, p. 4957-4961, 2005.

SHIH, C.C.; WU, Y.W.; LIN, W.C. Aqueous extract of Anoectochilus formosanus attenuate hepatic fibrosis induced by carbon tetrachloride in rats. Phytomedicine, v.12, p.453-460, 2005.

SNEDECOR, G.W.; COCHRAN, W.G. Statistical Methods. Iwoa: lowa State University Press, 1980. $75 p$.
TAVARES-DIAS, M; MORAES, F.R. Características hematológicas da Tilapia rendalli Boulenger, 1896 (osteichthyes: cichlidae) capturada em "pesquepague" de Franca, São Paulo, Brasil. Bioscience Journal, v.19, p.103-110, 2003.

TRACEY, K.J. Physiology and immunology of the cholinergic anti-inflammatory pathway. Journal of Clinical Investigation, v. 117, p.286-296, 2007.

YANG, Y.P; MA, X.M.; WANG,C.P. et al. Effect of increased hepatic platelet activating factor and its receptor portal hypertension in CCl4-induced liver cirrhosis. World Journal of Gastroenterology, v.12, n.5, p.709-715, 2006. 